Phys. Rev. Lett. 88 (2002) 192701

\title{
Probing the High Density Behaviour of Nuclear Symmetry Energy with High Energy Heavy-Ion Collisions
}

\author{
Bao-An Li* \\ Department of Chemistry and Physics \\ P.O. Box 419, Arkansas State University \\ State University, Arkansas 72467-0419, USA
}

\begin{abstract}
High energy heavy-ion collisions are proposed as a novel means to constrain stringently the high density (HD) behaviour of nuclear symmetry energy. Within an isospin-dependent hadronic transport model, it is shown for the first time that the isospin asymmetry of the HD nuclear matter formed in high energy heavyion collisions is uniquely determined by the HD behaviour of nuclear symmetry energy. Experimental signatures in two sensitive probes, i.e., $\pi^{-}$to $\pi^{+}$ratio and neutron-proton differential collective flow, are also investigated.
\end{abstract}

PACS numbers: 25.70.-z, 25.75.Ld., 24.10.Lx

*email: Bali@astate.edu 
The high density (HD) behaviour of nuclear symmetry energy $E_{\text {sym }}(\rho)$ is very important for understanding many interesting astrophysical phenomena, but it also subjects to the worst uncertainty among all properties of dense nuclear matter [1]. To study the HD behaviour of $E_{\text {sym }}(\rho)$ has been a longstanding goal of extensive research with various microscopic and/or phenomenological models over the last few decades, e.g., [2], for a recent review, see, e.g. [3]. The model predictions are extremely diverse and often contradictory. The results can be roughly classified into two groups, i.e., a group where the $E_{\text {sym }}(\rho)$ rises and one in which it falls with the increasing density. For instance, within the Hartree-Fock approach using about 25 Skyrme and Gony effective interactions that have been widely used successfully in studying saturation properties of symmetric nuclear matter and nuclear structures near the $\beta$ stability valley, the calculated symmetry energies were found to fall ap-

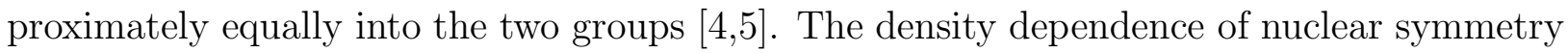
energy, especially at high densities, has many profound consequences for various studies in astrophysics [6,7]. In particular, an increasing $E_{\text {sym }}(\rho)$ leads to a relatively more proton-rich neutron star whereas a decreasing one would make the neutron star a pure neutron matter at high densities. Consequently, the chemical composition and cooling mechanisms of protoneutron stars [8,9], critical densities for Kaon condensations in dense stellar matter [10,11], mass-radius correlations [12,13] as well as the possibility of a mixed quark-hadron phase [14] in neutron stars will all be rather different. The fundamental cause of the extremely uncertain HD behaviour of $E_{\text {sym }}(\rho)$ is the complete lack of terrestrial laboratory data to constrain directly the model predictions. In this Letter, high energy heavy-ion collisions are proposed as a novel means to constrain stringently the HD behaviour of $E_{\text {sym }}(\rho)$. The upper bound of HD symmetry energy effects on high energy heavy-ion collisions is explored. It is shown for the first time that the neutron/proton ratio of HD nuclear matter formed in high energy heavy-ion collisions is uniquely determined by the HD behaviour of $E_{\text {sym }}(\rho)$. Experimental signatures of the HD behaviour of $E_{\text {sym }}(\rho)$ in two sensitive observables, i.e., the $\pi^{-} / \pi^{+}$ratio and the neutron-proton differential collective flow, are also investigated.

We use here the parabolic approximation of the equation of state (EOS) for isospin 
asymmetric nuclear matter [3]

$$
e(\rho, \delta)=e(\rho, 0)+E_{\text {sym }}(\rho) \delta^{2}
$$

where $e(\rho, 0)$ is the energy per nucleon in symmetric nuclear matter, and $\delta \equiv\left(\rho_{n}-\rho_{p}\right) /\left(\rho_{n}+\right.$ $\left.\rho_{p}\right)$ is the isospin asymmetry in terms of the neutron $\left(\rho_{n}\right)$ and proton $\left(\rho_{p}\right)$ density, respectively. For the isoscalar part we use the simplest, momentum-independent parameterization

$$
e(\rho, 0)=\frac{a}{2} u+\frac{b}{1+\sigma} u^{\sigma}+\frac{3}{5} e_{F}^{0} u^{2 / 3}
$$

where $u \equiv \rho / \rho_{0}$ is the reduced density and $e_{F}^{0}=36 \mathrm{MeV}$ is the Fermi energy. The parameters $a=-358.1 \mathrm{MeV}, b=304.8 \mathrm{MeV}$ and $\sigma=7 / 6$ are determined by saturation properties and a compressibility $K_{\infty}=201 \mathrm{MeV}$ of isospin symmetric nuclear matter. Our conclusions in this work are independent of the particular form of $e(\rho, 0)$. The symmetry energy is [1, 3 ,

$$
E_{\text {sym }}(\rho) \equiv e(\rho, 1)-e(\rho, 0)=\frac{5}{9} E_{k i n}(\rho, 0)+V_{2}(\rho)
$$

where $E_{k i n}(\rho, 0)$ is the kinetic energy per nucleon in symmetric nuclear matter and $V_{2}(\rho)$ is the deviation of the interaction energy of pure neutron matter from that of symmetric nuclear matter. The $E_{\text {sym }}(\rho)$ becomes negative if the condition $V_{2}(\rho) \leq-\frac{5}{9} E_{k i n}(\rho, 0)$ is reached at high densities. Thus a pure neutron matter could become most stable, leading to the isospin separation instability in HD neutron-rich matter. Consequently, pure neutron domains or neutron bubbles surrounding isolated protons can be formed in neutron stars [1]. To represent the two groups of model predictions, we use for the $E_{\text {sym }}(\rho)$

$$
E_{\text {sym }}^{a}(\rho) \equiv E_{\text {sym }}\left(\rho_{0}\right) u \text { and } E_{\text {sym }}^{b}(\rho) \equiv E_{\text {sym }}\left(\rho_{0}\right) u \cdot \frac{u_{c}-u}{u_{c}-1}
$$

where $E_{\text {sym }}\left(\rho_{0}\right)=30 \mathrm{MeV}$ is the symmetry energy at normal nuclear matter density, and $u_{c}=\rho_{c} / \rho_{0}$ is the reduced critical density at which the $E_{\text {sym }}^{b}(\rho)$ crosses zero and becomes negative at higher densities. The predicted value of $u_{c}$ ranges from about 2.7 (HartreeFock with the Skyrme interaction Sp [5]) to 9 (variational many-body approach with the UV14+UVII interaction [目].). These two forms of the $E_{\text {sym }}(\rho)$ (insert in the lower window) 
together with the corresponding EOS for isospin asymmetric nuclear matter are shown in Fig. 1. With the linearly increasing $E_{\text {sym }}^{a}(\rho)$, the EOS (upper window) becomes stiffer with the increasing $\delta$. The isospin symmetric nuclear matter remains to be the ground state at all densities. This is in stark contrast to the situation using the $E_{\text {sym }}^{b}(\rho)$. The EOS obtained with the $E_{\text {sym }}^{b}(\rho)$ and $u_{c}=3$ (lower window), is softened instead of being stiffened by the increasing isospin asymmetry $\delta$ at densities higher than $3 \rho_{0}$. At these high densities, the pure neutron matter and the isospin symmetric nuclear matter thus becomes the most stable and unstable form of HD nuclear matter, respectively.

High energy heavy-ion collisions provide the only terrestrial situation where the HD neutron-rich matter can be formed. Moreover, fast radioactive heavy-ion beams to be available at the planned Rare Isotope Accelerator (RIA) in the United States can make the HD nuclear matter even more neutron-rich. The HD behaviour of $E_{\text {sym }}(\rho)$ affects properties of the HD nuclear matter formed in high energy heavy-ion collisions. Moreover, it leads to interesting precursory phenomena in the experimental observables of high energy heavy-ion collisions. We investigate these effects and phenomena within an isospin-dependent hadronic transport model 15:16]. Evolutions of the phase-space distribution functions of nucleons, Delta resonances and pions with their explicit isospin degrees of freedom are solved numerically by using of the test-particle approach [17,18. Both the isoscalar and isovector mean-filed potentials are derived consistently from the nuclear EOS given above. Isospindependent total and differential cross sections among all particles are taken either from the elementary particle scattering data or obtained by using the detailed balance. Explicitly isospin-dependent Pauli blockings for fermions are also employed. For a review of the model, we refer the reader to ref. [19].

Shown in Fig. 2 is the correlation between the isospin asymmetry $\delta$ and the baryon density $\rho / \rho_{0}$ at the instant of $20 \mathrm{fm} / \mathrm{c}$ in the reaction of ${ }^{132} S n+{ }^{124} S n$ at a beam energy of $400 \mathrm{MeV} /$ nucleon and an impact parameter of $1 \mathrm{fm}$. This very reaction will be available at the RIA facility. The overall rise of $\delta$ at very low densities is due to the neutron skins of the 
colliding nuclei. One also notices that the $E_{\text {sym }}^{b}(\rho)$ leads to a slightly higher compression due to its relatively softening effect on the nuclear EOS. Dramatic effects due to the different symmetry energies are clearly revealed especially at high densities. For a comparison, the corresponding correlation in neutron stars at $\beta$ equilibrium is shown in the insert. As a reference, an estimate of the direct URCA limit of $\delta_{\beta}$ (below which the fast cooling process can happen in protoneutron stars and thus results in also higher neutrino flux) is indicated with the dotted line [8]. To a good approximation perfectly suitable for this study, a neutron star can be considered as consisting of nuetrons, protons and electrons. At $\beta$ equilibrium the proton fraction $x_{\beta} \equiv \rho_{p} /\left(\rho_{n}+\rho_{p}\right)$ in neutron stars is then determined by [8]

$$
\hbar c\left(3 \pi^{2} \rho x_{\beta}\right)^{1 / 3}=4 E_{\mathrm{sym}}(\rho)\left(1-2 x_{\beta}\right) .
$$

The equilibrium isospin asymmetry $\delta_{\beta}=1-2 x_{\beta}$ is therefore entirely determined by the $E_{\text {sym }}(\rho)$. With the $E_{\text {sym }}^{b}(\rho)$, the $\delta_{\beta}$ is 1 for $\rho / \rho_{0} \geq 3$, indicating that the neutron star has become a pure neutron matter at these high densities. On the contrary, with the $E_{\text {sym }}^{a}(\rho)$, the neutron star becomes so proton-rich that the direct URCA process can happen at densities higher than about $2.3 \rho_{0}$. An astonishing similarity is seen in the resultant $\delta-\rho$ correlations for the neutron star and the heavy-ion collision. In both cases, the symmetry energy $E_{\text {sym }}^{b}(\rho)$ makes the HD nuclear matter more neutron-rich than the $E_{\text {sym }}^{a}(\rho)$ and the effect grows with the increasing density. Of course, this is no surprise since the same nuclear EOS is at work in both cases. As shown in Fig. 1, in stark contrast to the situation with the $E_{\text {sym }}^{a}(\rho)$, the decreasing $E_{\text {sym }}^{b}(\rho)$ above $\rho_{0}$ makes it energetically more favorable to have the denser region more neutron-rich.

To further investigate the HD behaviour of $E_{\text {sym }}(\rho)$, the average $n / p$ ratio of the HD region with $\rho / \rho_{0} \geq 1$ is examined as a function of time and beam energy in the left panels of Fig. 3. The effect on $(n / p)_{\rho / \rho_{0} \geq 1}$ due to the different $E_{\text {sym }}(\rho)$ is seen to grow with both the reaction time and the beam energy. This is because of the higher densities reached with the more energetic beams and after longer times of compression.

How to probe experimentally the HD behaviour of $E_{\text {sym }}(\rho)$ in high energy heavy-ion 
collisions? Among the observables we have explored, the $\pi^{-} / \pi^{+}$ratio and the neutronproton differential collective flow are found to be most promising. The former measures sensitively the neutron to proton ratio $n / p$ of the HD nuclear matter and thus indirectly the HD behaviour of $E_{\text {sym }}(\rho)$. At beam energies below about $2 \mathrm{GeV} /$ nucleon, pions are mostly produced through the decay of $\Delta(1232)$ resonances. The primordial $\pi^{-} / \pi^{+}$ratio is approximately quadratic in $\mathrm{n} / \mathrm{p}$ according to the branching ratios of single pion production via $\Delta$ resonances in nucleon-nucleon collisions

$$
\pi^{-} / \pi^{+}=\frac{5 n^{2}+n p}{5 p^{2}+n p} \approx(n / p)^{2}
$$

Pion reabsorptions and rescatterings are expected to complicate the above relationship. Nevertheless, very high sensitivity to the $n / p$ ratio is retained in the final $\pi^{-} / \pi^{+}$ratio as indicated in the experimental data of high energy heavy-ion collisions [20]. Shown in the right panels of Fig. 3 are the $\left(\pi^{-} / \pi^{+}\right)_{\text {like }}$ ratio

$$
\left(\pi^{-} / \pi^{+}\right)_{l i k e} \equiv \frac{\pi^{-}+\Delta^{-}+\frac{1}{3} \Delta^{0}}{\pi^{+}+\Delta^{++}+\frac{1}{3} \Delta^{+}}
$$

as a function of time. This ratio naturally becomes the final $\pi^{-} / \pi^{+}$ratio when the reaction time $t$ is much longer than the lifetime of the delta resonance $\tau_{\Delta}$. The $\left(\pi^{-} / \pi^{+}\right)_{\text {like }}$ ratio is rather high in the early stage of the reaction because of the large numbers of neutron-neutron scatterings near the surfaces of the colliding nuclei. By comparing the two sides in Fig. 3, it is seen that a variation of about $30 \%$ in the $(n / p)_{\rho / \rho_{0} \geq 1}$ ratio due to the different $E_{\text {sym }}(\rho)$ results in about $15 \%$ change in the final $\pi^{-} / \pi^{+}$ratio. The later thus has an appreciable response factor of about 0.5 to the variation of $\mathrm{HD} \mathrm{n} / \mathrm{p}$ ratio, and it is approximately beam energy independent.

The neutron-proton differential collective flow is measured by 21

$$
F_{n p}(y) \equiv \frac{1}{N(y)} \sum_{i=1}^{N(y)} p_{x_{i}} \tau_{i}
$$

where $N(y)$ is the total number of free nucleons at the rapidity $y, p_{x_{i}}$ is the transverse momentum of particle $i$ in the reaction plane, and $\tau_{i}$ is +1 and -1 for neutrons and protons, 
respectively. The $F_{n p}(y)$ combines constructively the in-plane transverse momenta generated by the isovector potentials while reducing significantly influences of the isoscalar potentials of both neutrons and protons. Thus, it can reveal more directly the HD behaviour of $E_{\text {sym }}(\rho)$ in high energy heavy-ion collisions. A typical result for the ${ }^{132} S n+{ }^{124} S n$ reaction is shown in the upper window of Fig. 4. A clear signature of the HD behaviour of $E_{\text {sym }}(\rho)$ appears at both forward and backward rapidities. To characterize the effect, the slope $d F_{n p} / d\left(y_{c m} / y_{\text {beam }}\right)$ at mid-rapidity is shown as a function of beam energy in the lower window. A striking difference of about a factor of 2 exists in the reactions at $E_{\text {beam }} \geq 200 \mathrm{MeV} /$ nucleon. This large effect can be very easily observed by using available detectors at several heavy-ion facilities in the world 22,23]. Compared with the $\pi^{-} / \pi^{+}$ratio, the neutron-proton differential collective flow is more directly affected by and is thus also a more sensitive probe of the HD behaviour of $E_{\text {sym }}(\rho)$.

In conclusion, the HD behaviour of $E_{\text {sym }}(\rho)$ has been puzzling physicists for decades. In this work, high energy heavy-ion collisions are proposed as a novel means to solve this longstanding problem. The upper bound of HD symmetry energy effects on high energy heavy-ion collisions is explored. For the first time, it is shown that the isospin asymmetry of HD nuclear matter formed in high energy heavy-ion collisions is uniquely determined by the HD behaviour of $E_{\text {sym }}(\rho)$. Both the $\pi^{-} / \pi^{+}$ratio and the neutron-proton differential collective flow can serve as sensitive probes. Measurements of these observables will provide the first terrestrial data to constrain stringently the HD behaviour of nuclear symmetry energy.

This work was supported in part by the National Science Foundation Grant No. PHY0088934 and Arkansas Science and Technology Authority Grant No. 00-B-14. 


\section{REFERENCES}

[1] M. Kutschera, Phys. Lett. B340, 1 (1994); Z. Phys. A348, 263 (1994); Acta Phys. Polon. B29, 25 (1998).

[2] K.A. Brueckner, S.A. Coon and J. Dabrowski, Phys. Rev. 168, 1184 (1967); P.J. Siemens, Nucl. Phys. A141, 225 (1970); V.R. Pandharipande et al., Phys. Lett. B38, 608 (1972); B. Friedman et al., Nucl. Phys. A361, 502 (1981); I.E. Lagaris et al., Nucl. Phys. A369, 470 (1981); S.A. Chin, Ann. Phys. (N.Y.), 108, 301 (1977); B.D. Serot and J.D. Walecka, Adv. Nucl. Phys. 16, 1 (1986); B. ter Haar and R. Malfliet, Phys. Rev. Lett. 59, 1652 (1987); H. Müther, M. Prakash and T.L. Ainsworth, Phys. Lett. B199, 469 (1987); R.B. Wiringa, V. Fiks and A. Fabrocini, Phys. Rev. C38, 1010 (1988); I. Bombaci and U. Lombardo, Phys. Rev. C44, 1892 (1991); A. Akmal, V.R. Pandharipande and D.G. Ravenhall, Phys. Rev. C58, 1804 (1998); H. Huber, F. Weber, and M.K. Weigel, Phys. Rev. C57, 3484 (1998); W.D. Myers and W.J. Światecki, Phys. Rev. C57, 3020 (1998); C.-H. Lee, T.T.S. Kuo, G.Q. Li and G.E. Brown, Phys. Rev. C57, 3488 (1998).

[3] I. Bombaci, Chapter 2 in Isospin Physics in Heavy-Ion Collisions at Intermediate Energies, Eds. Bao-An Li and W. Udo Schröder, ISBN 1-56072-888-4, Nova Science Publishers, Inc (2001, New York).

[4] B.A. Brown, Phys. Rev. Lett. 85, 5296 (2000).

[5] J. Margueron, J. Navarro, N. Van Giai and W. Jing, nucl-th/0110026.

[6] J.M. Lattimer and M. Prakash, Astrophys J. 550, 426 (2001).

[7] M. Prakash et al., Phys. Rep. 280, 1 (1997).

[8] J.M. Lattimer et al., Phys. Rev. Lett. 66, 2701 (1991).

[9] K. Sumiyoshi and H. Toki, Astro. Phys. Journal, 422, 700 (1994). 
[10] C.-H. Lee, Phys. Rep. 275, 255 (1996) and references therein.

[11] S. Kubis and M. Kutschera, Acta Phys. Polon. B30, 2747 (1999).

[12] M. Prakash, T.L. Ainsworth and J.M. Lattimer, Phys. Rev. Lett. 61, 2518 (1988).

[13] L. Engvik et al., Phys. Rev. Lett. 73, 2650 (1994).

[14] M. Kutschera and J. Niemiec, Phys. Rev. C62, 025802 (2000).

[15] B.A. Li and W. Bauer, Phys. Rev. C44, 450, (1991).

[16] B.A. Li et al., Phys. Rev. Lett. 76, 4492 (1996); 78, 1644 (1997); 82, 5004 (1999).

[17] C.Y. Wong, Phys. Rev. C25, 1461 (1982).

[18] G.F. Bertsch and Subal Das Gupta, Phys. Rep. 160, 189 (1988).

[19] B.A. Li, C.M. Ko and W. Bauer, topical review, Int. Jou. Mod. Phys. E7, 147 (1998).

[20] R. Stock, Phys. Rep. 135, 259 (1986).

[21] B.A. Li, Phys. Rev. Lett. 85, 4221 (2000).

[22] W.G. Lynch, private communications.

[23] G.D. Westfall et al., Phys. Rev. Lett. 71, 1986 (1993). 


\section{FIGURES}

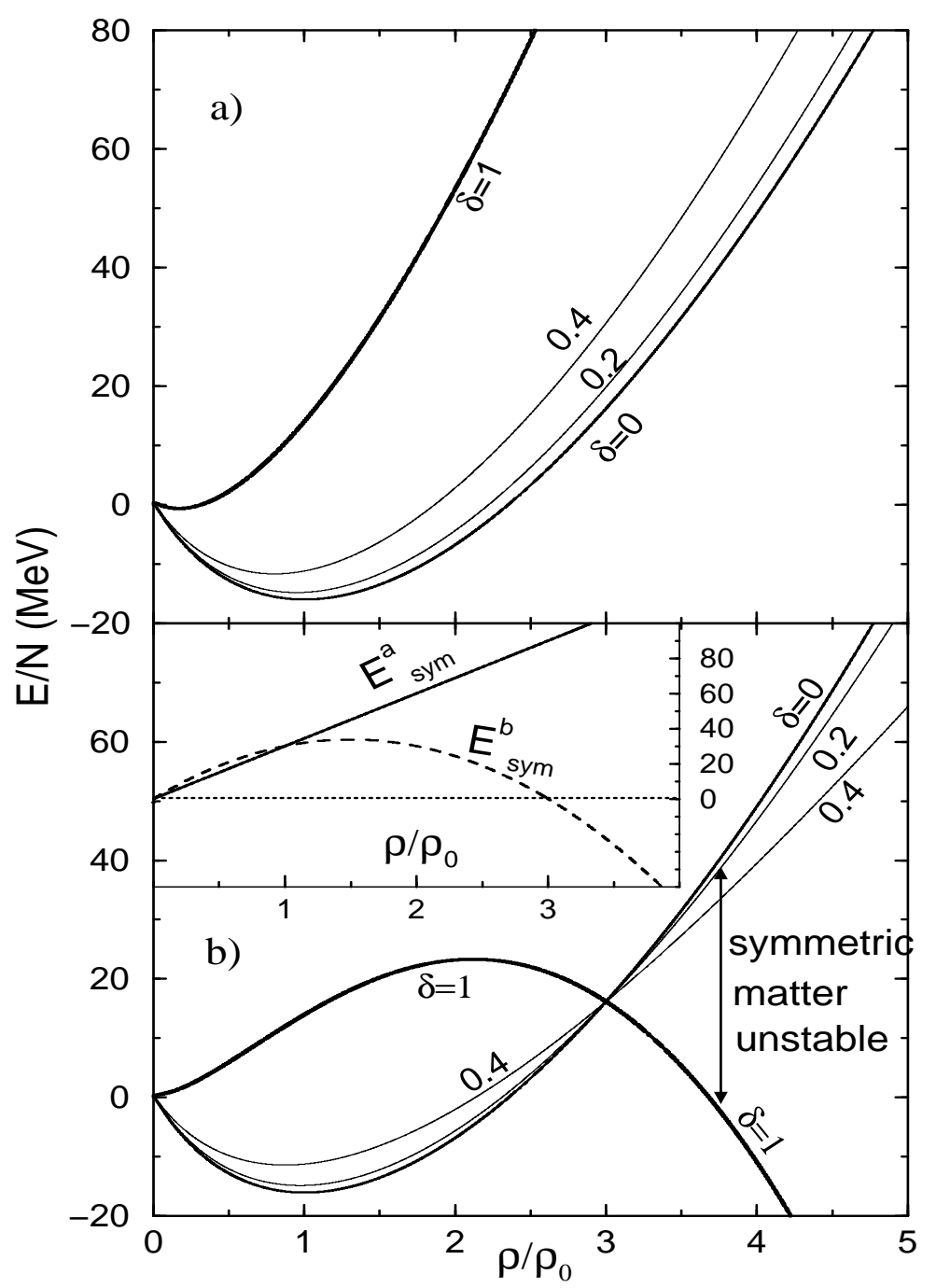

FIG. 1. The upper (lower) window is the equation of state of isospin-asymmetric nuclear matter corresponding to the nuclear symmetry energy parameterization $E_{\text {sym }}^{a}\left(E_{\text {sym }}^{b}\right)$ shown in the insert of the lower window. 


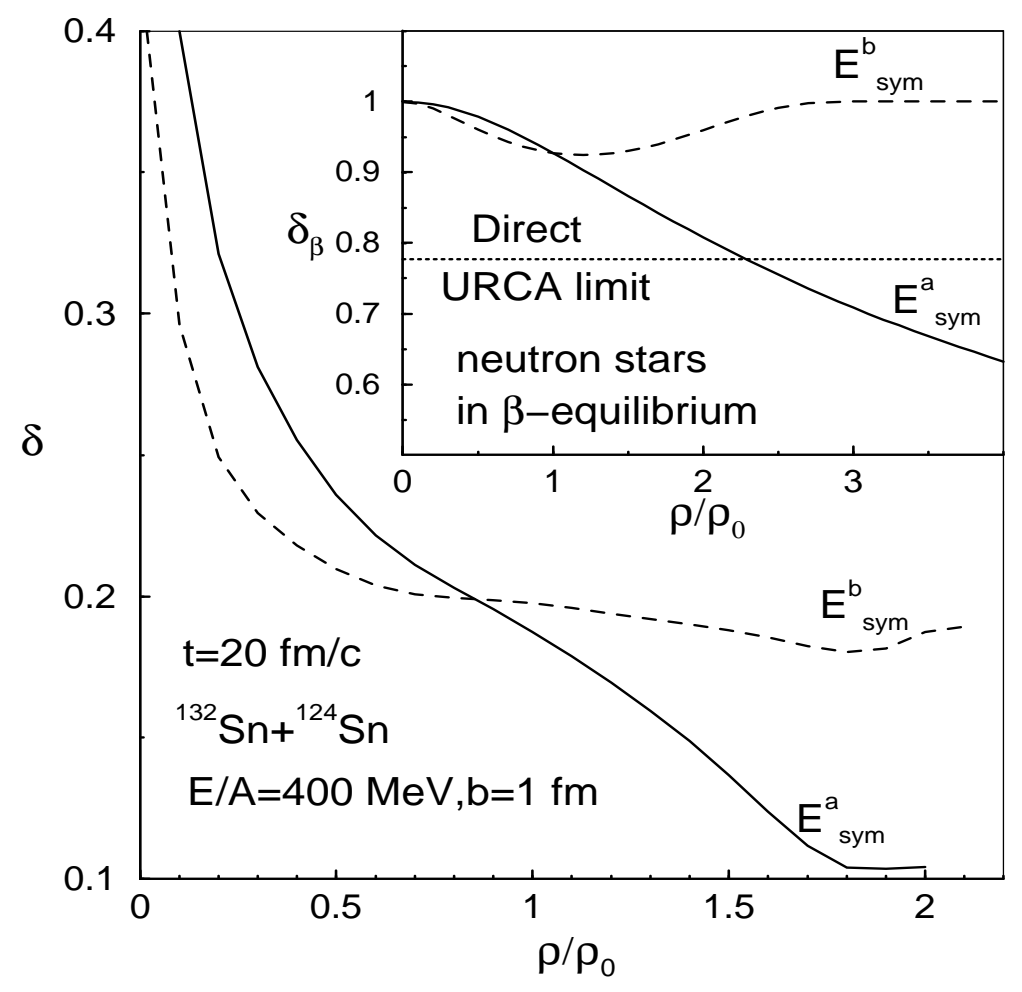

FIG. 2. The isospin asymmetry-density correlation at $\mathrm{t}=20 \mathrm{fm} / \mathrm{c}$ over the whole space in the ${ }^{132} S n+{ }^{124} S n$ reaction with the nuclear symmetry energy $E_{\text {sym }}^{a}$ and $E_{\text {sym }}^{b}$, respectively. The corresponding correlation in neutron stars is shown in the insert. 


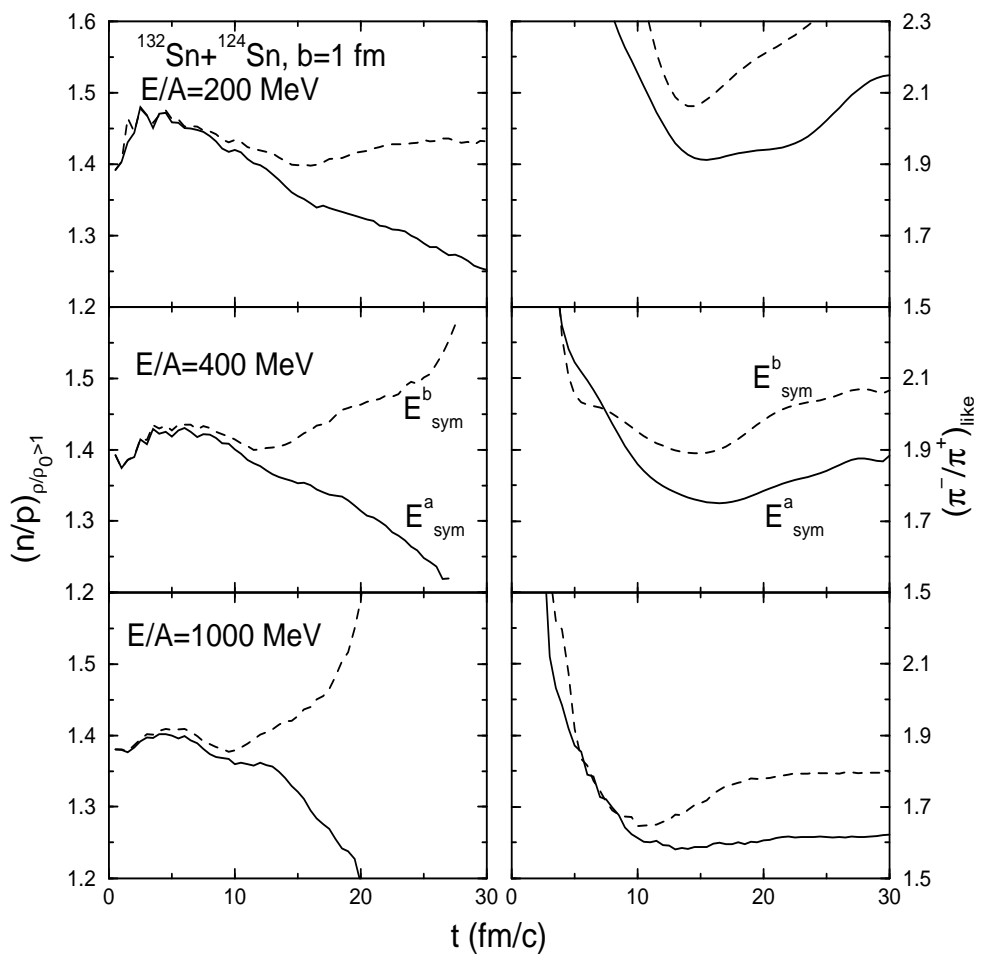

FIG. 3. Left panels: the neutron/proton ratio of nuclear matter with density higher than normal nuclear matter density as a function of time with the nuclear symmetry energy $E_{\text {sym }}^{a}$ and $E_{\text {sym }}^{b}$, respectively. Right panels: $\left(\pi^{-} / \pi^{+}\right)_{l i k e}$ ratios in the same reactions. 

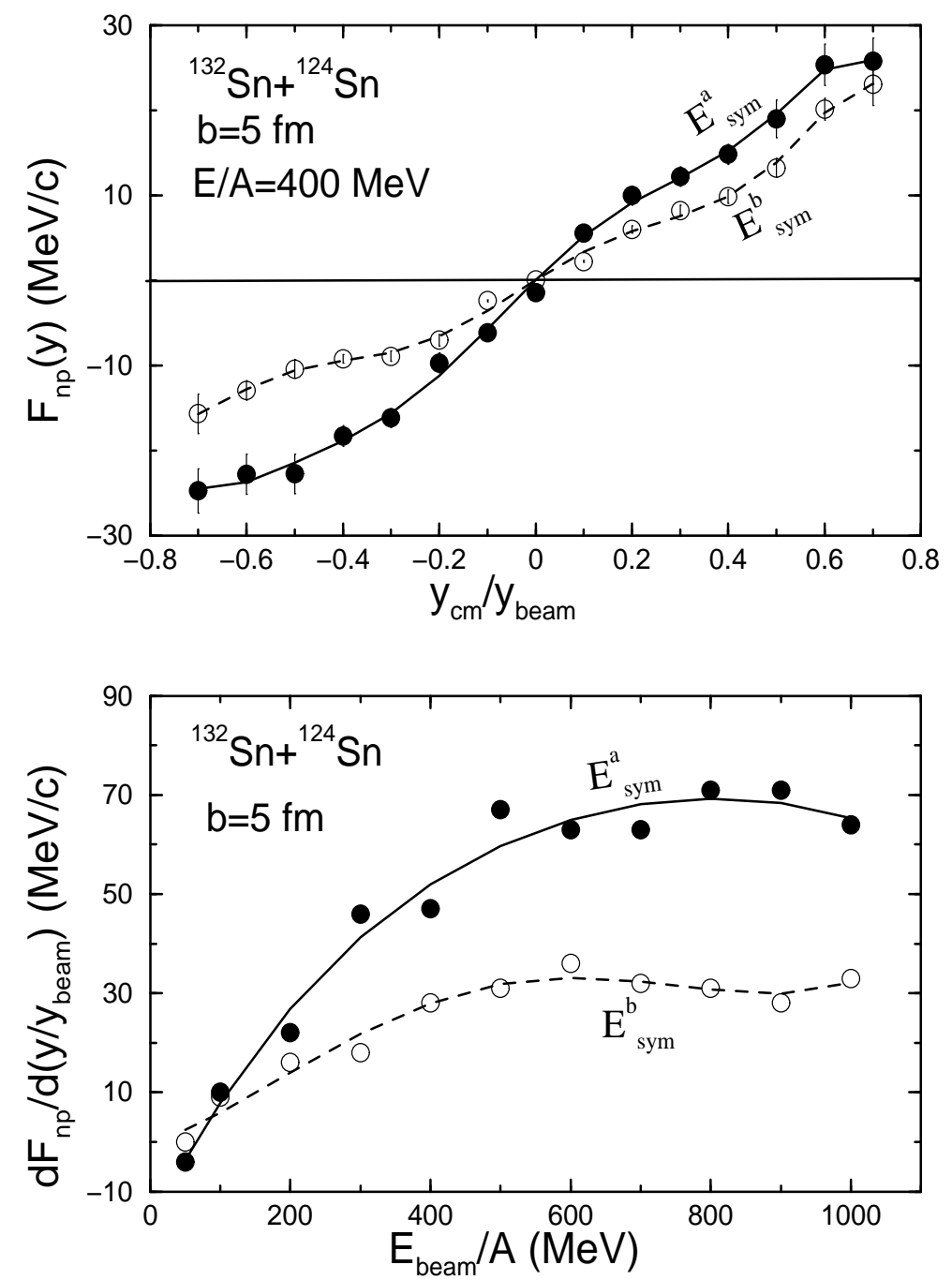

FIG. 4. Upper window: the neutron-proton differential collective flow in the ${ }^{132} S n+{ }^{124} S n$ reaction with the nuclear symmetry energy $E_{\text {sym }}^{a}$ and $E_{\text {sym }}^{b}$, respectively. Lower window: excitation function of the slope parameter of the differential flow for the ${ }^{132} S n+{ }^{124} S n$ reaction. The solid (with the $E_{\text {sym }}^{a}$ ) and dashed (with the $E_{\text {sym }}^{b}$ ) lines are drawn to guide the eye. 